\title{
Analysis of Phenomena in Safety Systems Made of Hyper-Elastic Materials - Selected Issues
}

\author{
Michał FOTEK ${ }^{1}$, Łukasz GOŁĘBIEWSKI ${ }^{2}$, Jarosław MAŃKOWSKI ${ }^{3}$ and \\ Piotr $\dot{\mathrm{Z} A C H}{ }^{4}$ \\ Institute of Machine Design Fundamentals, Faculty of Automotive and Construction \\ Machinery Engineering, Warsaw University of Technology \\ Narbutta 84, 02-524 Warsaw, Poland
}

\begin{abstract}
The article describes the process of experience building and knowledge shaping on the example of the study of phenomena occurring in the structure of polymeric safety buffers. A real example has been used as a normative qualification criterion allowing the security team to operate. The analysis of physico-mechanical properties of non-linear polymeric material of cellular structure was performed by considering the proposed mathematical description of the issues, based on experimental bench tests and using computer numerical methods. The work is devoted to extending the knowledge of processes occurring during critical interactions in safety systems based on polymer structures. To develop a methodology for identification of phenomena and design of products dedicated to minimizing the effects of accidents. Propose modern methods of verification and selection of passive safety systems. The conducted works were of a multi-criteria and multidisciplinary character guaranteeing direct translation of results and use of effects in other engineering techniques.
\end{abstract}

Keywords. polymer buffering units, hyperelasticity, material damping

\section{Introduction}

Energy-absorbing elements are used to limit the effects of dynamic loads created by an emergency. Made of hyper-elastic materials, they are used among others in cars (as elements of suspensions and bumpers), aircraft landing gears, as well as in vertical transport systems. In the design process, the analysis of necessary issues requires considering many different phenomena, such as: plastic deformation, cracking, or energy dissipation due to friction.

The methodology of structural design, particularly for the aviation and automotive industries, is based on the use of new materials: plastics and composites, as alternatives to traditional materials. Modifications of structures to minimize the effects of failures, accidents, collisions are carried out to achieve the highest possible energy absorption at low system weight. [1, 2]

${ }^{1}$ Corresponding author, Mail: michal.fotek@pw.edu.pl

${ }^{2}$ Corresponding author, Mail: lukasz.golebiewski@pw.edu.pl

${ }^{3}$ Corresponding author, Mail: jaroslaw.mankowski@pw.edu.pl

${ }^{4}$ Corresponding author, Mail: piotr.zach@pw.edu.pl 
The processes required for consideration are complex and often highly non-linear in nature. For this reason, it is difficult to assess the behaviour of an energy-intensive element. Design processes aimed at producing a system from new materials, e.g. polymers, complicate the issues. It is required to use cognitive, research and analytical engineering techniques of more than subject matter character.

The article describes the process of experience building and knowledge shaping on the example of the study of phenomena occurring in the structure of polymeric safety buffers. A real example has been used as a normative qualification criterion allowing the security team to operate. Critical case of passenger lift cabin failure, in the context of passenger safety and lift structure strength, was considered by analysing the elastomeric safety unit. The analysis of physico-mechanical properties of non-linear polymeric material of cellular structure was performed by considering the proposed mathematical description of the issues, based on experimental bench tests and with the use of computer numerical methods. The influence of load history, structure degradation and load velocity on changes in buffer operation character were investigated.

The aim of the verification was to extend the knowledge of processes occurring during critical interactions in safety systems based on polymer structures, to develop a methodology for identification of phenomena and design of products dedicated to minimizing the effects of accidents and improving the safety of people and equipment, to develop modern methods of verification and selection of passive safety systems. The conducted works were of multi-criteria and multidisciplinary character guaranteeing direct translation of results and use of effects in other engineering techniques.

\section{Characteristics of polymer buffering units}

Safety buffers are a safety element required in a passenger crane assembly according to european standard [3]. The purpose of using the unit is to protect the crane and passengers from the effects of the cabin exceeding the permissible lower position and hitting the elements located in the elevator pit.

An incident when the cabin overruns the bumper is a danger to both the passengers and the structure of the crane. Kinetic energy of a moving cabin about mass $950 \mathrm{~kg}$, falling freely from a height $1 \mathrm{~m} \mathrm{~kg}$ had to be dispersed by the bumper on a distance 0.1 $\mathrm{m}$. Therefore, high dynamic loads are generated.

Buffer bumper solutions can be divided according to the following work characteristics: linear (spring), energy dissipating (hydraulic), energy absorbing and dissipating, non-linear (elastomer).

Foamed materials are used for elastic and damping elements in safety systems for cranes, vehicles and as machine foundation systems. The use of polyurethane structures is supported by the ease of designing favourable physico-mechanical properties, technology and low price of products. PUR is a thermoplastic material obtained by polyaddition of aromatic or aliphatic diisocyanates with compounds containing at least two hydroxyl groups, e.g. diols or polyethers. Different properties of the materials obtained are determined by the isocyanates and polyols contained in them.

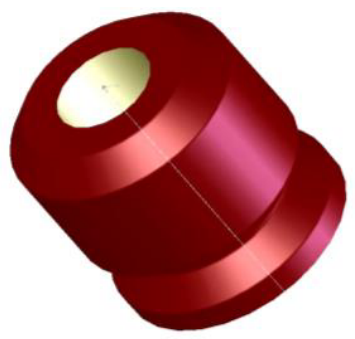

Figure 1. The tested buffer of the company EN2 ETN GmbH. 
The materials used to build elastomeric energy-dissipating buffers are foamed polyurethanes such as Cellasto ${ }^{\circledR}(\mathrm{BASF})$, Diepocell ${ }^{\circledR}(\mathrm{P}+\mathrm{S}$ Polyurethan-Elastomere $\mathrm{GmbH} \&$ Co. KG), AUTAN® (ACLA-WERKE GmbH), Cell-PU (ETN GmbH). The shape of the tested buffers are presented on figure 1.

\section{Analysis of phenomena in a system with an elastomeric element}

Using the classic description of a machine assembly subjected to loads in the form of vibrations extended by the performance characteristics of polyurethane elastomers [4-6] the description of phenomena occurring during the operation of buffer assemblies is described by a mathematical model reduced to the mass of the machine $m$, according to the scheme shown in figure 2.

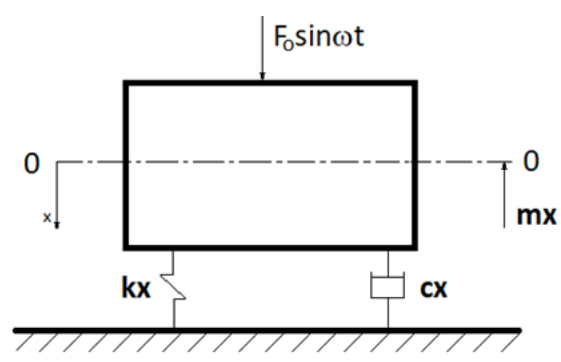

Figure 2. Diagram of the vibration system.

The layout shown can be described with the use of the equation (1):

$$
m x^{\prime \prime}+c x^{\prime}+k x=F_{0} \sin \sin \omega t
$$

where: $m x^{\prime \prime}$ - inertia, $c x^{\prime}$-internal friction force, $k x$ - restoring force.

Internal friction is determined by the mechanical loss factor d i.e. tan angle tan $\delta$. Tangents $\delta$ is the angle between the source deflection arrow and the final deformation. (2):

The properties of the mounting springs affect the natural frequency of the system

$$
\omega_{r z}=\sqrt{\frac{c}{m}}
$$

where:

$$
\begin{aligned}
& c=\frac{F}{X_{f}} \\
& \omega=2 \pi n
\end{aligned}
$$

If the elastic deflection $X_{f}$ is in [mm] based on equation (3) it is possible to designate critical frequency $n$ in $[\mathrm{Hz}]$. Internal friction and restoring force determine the size of resonant frequency and the increase in $V_{D}$ amplitude. Dimensionless $V_{D}$ value is the solution of equation (1). In a mounting system, the system's response force can be estimated according to dependency (5):

$$
V_{D}=\frac{F_{\text {reszt. }}}{F_{\text {dost. }}}=\frac{1+j d \lambda}{1-\lambda^{2}+j d \lambda}
$$


$V_{D}$ should be considered in the selection of the protective elements. Damping coefficient of the material determines the effectiveness of vibration damping, which for the system under consideration can be determined from dependency (6).

$$
\eta_{D}=\left(100-V_{D}\right)
$$

The desired system damping performance (as high as possible) can only be achieved with a low force ratio $F_{\text {dost }} i F_{\text {reszt. }}$ i.e. a small coefficient $\left.V_{D} \rightarrow \lambda\right\rangle \sqrt{2}, d \approx 0$.

Based on the results obtained, the natural vibration frequency for the critical load case and the desired damping performance of the system were determined. The model was used for selection and commercial selection of the polyurethane elastomeric protection unit for research works. It was assumed that the element should be characterized by the lowest possible mechanical loss factor d, while low natural frequencies were achieved.

\section{Experimental Research}

The buffer EN2 was made out of poliuretan with the trade name PUR Cellasto from the company ETN GmbH. In order to determine the physical and mechanical properties, basic tests of the elastomeric material were performed. The tests were performed in accordance with the guidelines of the european standard [3]. For the tests the specimens, cut out of the buffer: rectangular beams (static tensile tests), cubic cubes (static compression tests) were used.

It has been assumed [2], that there is an asymmetric characteristic of operation of PUR foam elements in compression and tension. Therefore, the experiments included static tension and compression, in range of strain up to $80 \%$ of nominal strain, and free compression at speeds: 0.1 $\mathrm{mm} / \mathrm{s}, 1 \mathrm{~mm} / \mathrm{s}, 10 \mathrm{~mm} / \mathrm{s}, 40 \mathrm{~mm} / \mathrm{s}$ in the range from $10 \%$ to $80 \%$ of nominal strain. Figure 3 shows the characteristics of $\sigma(\varepsilon)$ PUR material.

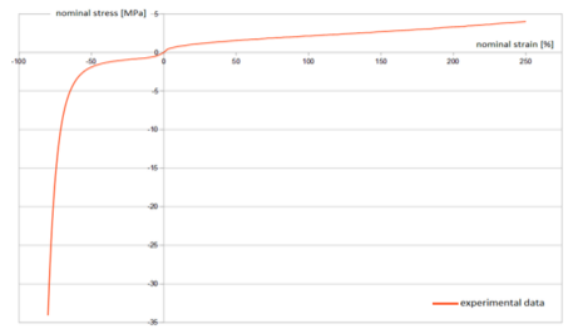

Figure 3. Characteristics of nominal axial stresses as a function of axial strain of specimens cut out of buffers [8].

Figure 4 shows the registered tensile (a) and compressive (b) tests. Cellasto PUR characteristic values were used for numerical identification of material in FEM systems and preparation of functional models of buffer units.

a)

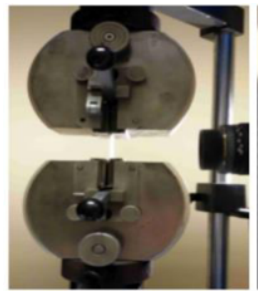

b)

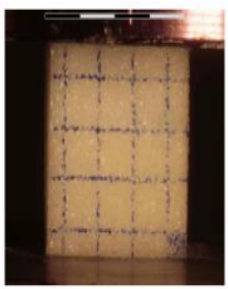

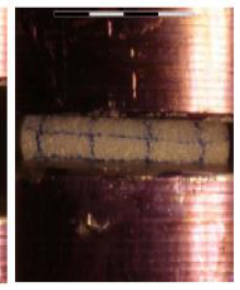

Figure 4. Executing experimental tests [8]. 
The polyurethane from which the buffer is made has a cellular structure. In order to characterize the structure of the material, tests were performed using optical and scanning microscopy. It was found that the sizes of spherical, open pores are uneven in the entire buffer volume. They depend on the course of foaming during production. The pore diameter ranges from 0.1-0.2 mm for less and 0.4-1 mm, for more foamed areas. The resultant density of the tested material is $0.5 \mathrm{~g} / \mathrm{cm} 3$.

The nominal strain curves obtained from the compression tests are shown in figure 5. Comparing the obtained characteristics of the new and tested material, it was found that the material with a load history has a lower stiffness in both compression and tensile (figure 6). The evaluation of changes at the level of PUR material structure and mechanical properties resulting from the deformation of the buffer element during operation was performed. The verification was based on experimental tests and microscopic observations.

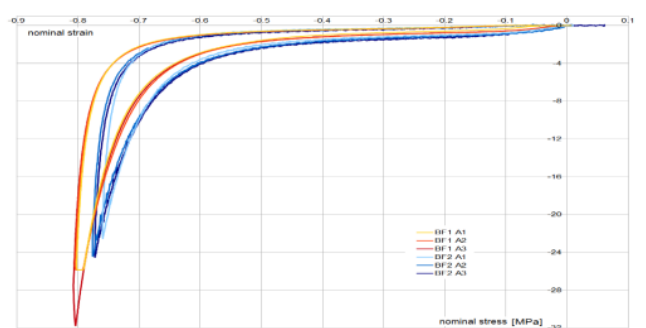

Figure 5. Characteristics of nominal axial stresses as a function of axial strain of specimens cut out of buffers [8].

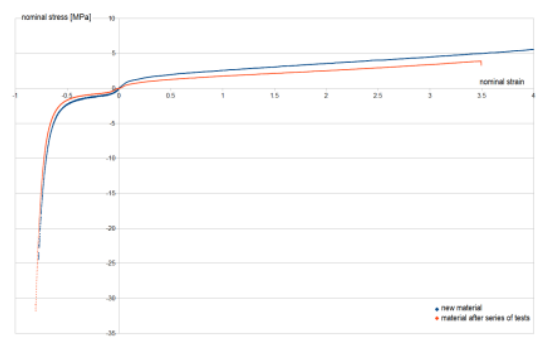

Figure 6. Characteristics of nominal axial stress as a function of axial strain for new material and with strain history [8].

External evaluation did not indicate any permanent changes or buffer damage. After cutting into smaller samples, damage to the material structure was noticed on the inner cylindrical surface of the buffer.

An evaluation of behaviour of the physical buffer element at speeds: $0.1 \mathrm{~mm} / \mathrm{s}$, $1 \mathrm{~mm} / \mathrm{s}, 10 \mathrm{~mm} / \mathrm{s}, 40 \mathrm{~mm} / \mathrm{s}$ in the range from $10 \%$ to $80 \%$ of nominal strain was carried out. In order to assess the effect of the relaxation phenomenon [2], the tests were carried out in three rounds, at an interval of two weeks each. Figure 7 shows an example of the implementation of the test.

a)

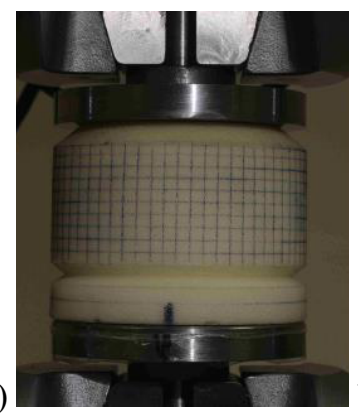

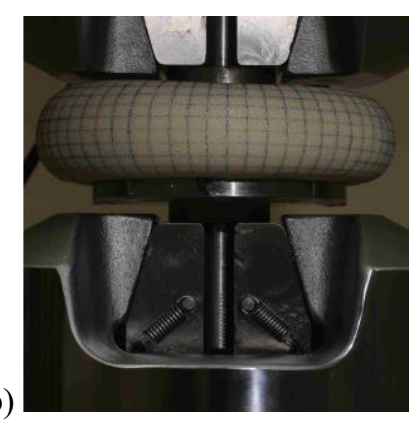

Figure 7. Buffer a) before the experiment b) during the experiment - nominal strain $80 \%$ [8].

The phenomenon of stress relaxation in the material structure was observed. Figure 8 shows the time course of the buffer compression test. It should be noted that despite maintaining a constant position of the machine head support, the axial force decreases, which suggests that there are changes in the stress condition of the specimen. 
The recorded loading and unloading characteristics are arranged in hysteresis loops, the field of which indicates the amount of energy dissipated by the energy element under investigation. The study did not consider the issue of internal damping, but it is worth noting that in one full cycle of deformation, the field of hysteresis loops represents up to $60 \%$ of total deflection energy. Figure 9 shows the change of buffer deformation energy during loading and unloading obtained by integration of the axial force course in relation to displacement.

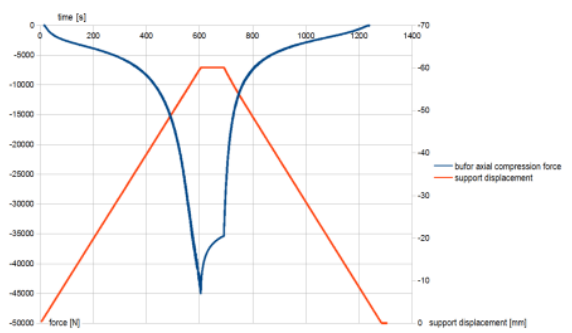

Figure 8. The course of the buffer compression test over time [8].

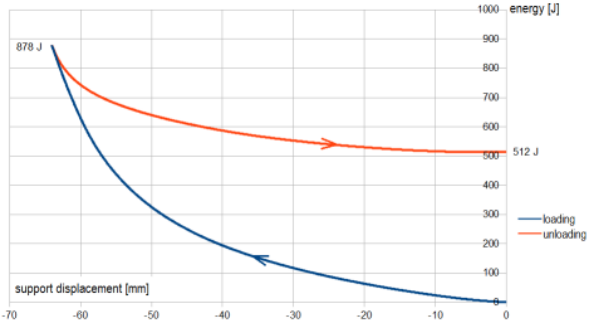

Figure 9. Deformation energy of the buffer - the direction of the course in time was indicated [8].

\section{Model of material}

Different models of hyperelastic materials are described in the literature. Each of them has its own characteristics and was created to describe selected materials [9-20]. For the purposes of the study, the assumptions of the material models were analysed and the results of the research were discussed. The calculation of model coefficients was based on the tabular representation of the material curve, created on the basis of experimental results shown in figure 3. The models proposed by Ray W. Ogden in 1972 and R. Hill and B. Storåkers' so-called 'hyperfoam' model were considered.

Among the considered models the Hill-Storakers model, which is a modified Ogden model, proposed by R. Hill and B. Storakers for the needs of modeling isotopic foamed materials. The model assumes that the behaviour of the material during deformation can be described by a function of the form deformation density and on this basis the relationship between stresses and deformations can be derived. In this case the deformation energy density is described with the dependence (7) [21][22]:

$$
W=\sum_{i=1}^{n} \frac{2 \mu_{i}}{\alpha_{i}^{2}}\left[\hat{\lambda}_{1}^{\alpha_{i}}+\hat{\lambda}_{2}^{\alpha_{i}}+\hat{\lambda}_{3}^{\alpha_{i}}-3+\frac{1}{\beta_{i}}\left(J_{e l}^{-\alpha_{i} \beta_{i}}-1\right)\right]
$$

where:

$\lambda_{1}, \lambda_{2}, \lambda_{3}-$ the deviatoric stretches,

$\mathrm{J}^{\mathrm{el}}-$ the elastic volume ratio,

$\mu_{\mathrm{i}}-$ are coefficients related to the initial shear modulus,

$\beta \mathrm{i}-$ are coefficients related to the Poisson's ratio.

It appears from the considerations made that the third degree Hyperfoam model based on coefficients according to Table 1 best represents the behaviour of the material. It provides both greater stability of calculations and better adjustment to experimental data at higher deformations. The limitation was the stability of the analyses due to high local stresses in the contact areas. Plastic deformations in the model were allowed. The 
yield strength was determined from the values of permanent deformation from the compression tests. Figure 10 shows the relationship between stress and plastic deformation implemented for the verified material models. The results of the evaluation of the Hill-Straker's model of 3rd degree - Hyperfit are shown in figure 11. The parameterized description of the model was used in numerical analyses.

Table 1. Hill-Storakers model coefficients for degree $n=3$.

\begin{tabular}{|c|c|c|c|}
\hline $\mathbf{i}$ & $\boldsymbol{\mu}_{\mathbf{i}}$ & $\boldsymbol{\alpha}_{\mathbf{i}}$ & $\boldsymbol{\beta}_{\mathbf{i}}$ \\
\hline 1 & -1.6925918891 & 0.00263931423 & -0.213821998 \\
\hline 2 & 2.3606046565 & 1.519090909993 & 0.2676923057 \\
\hline 3 & 0.2411390007 & -2.23355096391 & -0.0882572061 \\
\hline
\end{tabular}

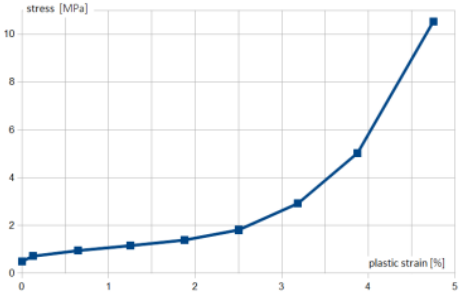

Figure 10. Plastic strain characteristic defined for researched material models [8].

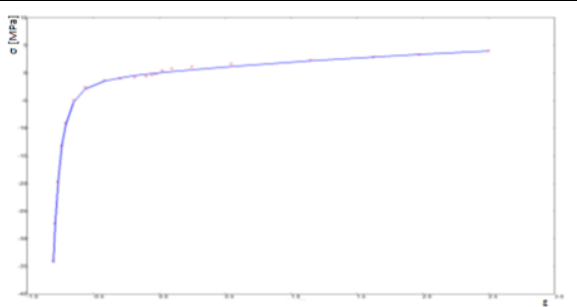

Figure 11. Results of evaluation Hill-Storakers $3^{\text {rd }}$ degree model - Hyperfit [8].

\section{FEM model analyses}

The curves of the material tests were used in a similar tensile test in a virtual environment. Materials with a maximum engineering strain like several hundred percent require the use of a simulation curve of true stress - true strain. Using the stresses and deformations referenced to the initial section. True strain calculated according to the equation (8). True stress received directly from the uniaxial tensile test as the ratio of instantaneous force and cross-section sample.

$$
\varepsilon_{\text {real }}=\frac{l_{0}+\Delta l}{l_{0}}=\ln \left(1+\varepsilon_{\text {eng }}\right)
$$

Tensile simulations of PUR samples were performed. Curve true stress - true strain obtained from the simulation was compared with input curve, until convergence has been obtained. They allowed for the selection of the appropriate material model and selecting the appropriate description of the material curve. Figure 12 shows the simulation results.

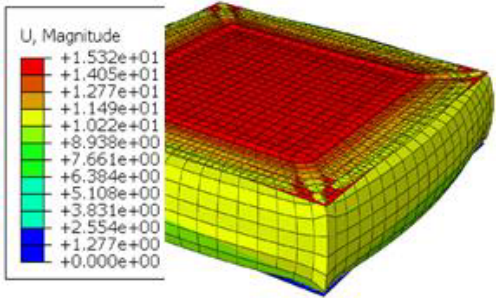

Figure 12. PUR test by Hyperfoam model - FEM (reverse engineering). a)

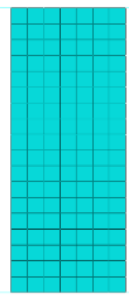

b)

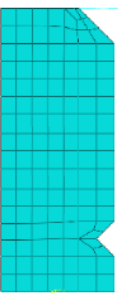

Figure 13. Buffer models used for analysis: a) simplified model, b) precise model. 
The buffer work was analysed using two different geometric models. The first one was a simplified model, the second one included two peripheral undercuts. The axial symmetry of the buffer was used to perform analyses in two-dimensional space. The buffer cross-section model is placed between the elements reflecting the pressure plates. A rigid frictional contact was defined between the plates and the analysed unit. Fournode hybrid elements were used for cross-section analysis. Edge conditions were defined in the symmetry axis of both clamping elements. The load was given by linearly increasing displacement of the upper clamping element until a deflection of $80 \%$ was achieved.

It was found that the developed model of the material allows for mapping of the global buffer deformation. The buffer compression characteristics obtained by numerical analyses show deviations from experimental observations in the range of material expansion beyond the compression elements. The differences found were a consequence of the adopted approximations in mapping the material behavior. A comparison of the deformation of the actual object with the FEM model is shown in Figure 14. For better illustration, the results from the axisymmetric model are displayed in a three-dimensional form.

a)

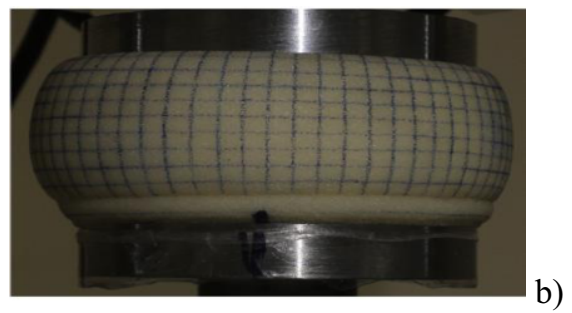

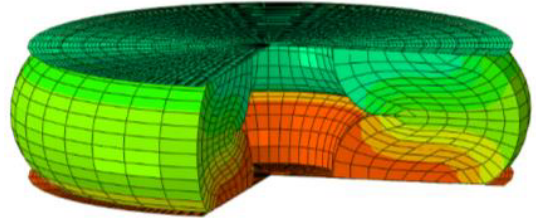

b)

Figure14. Comparison of the results of the buffer tests (a) with the results of the numerical simulation (b).

\section{Summary}

It has been proved that it is necessary to recognise and describe the phenomena occurring in the structure of the material used to manufacture safety systems and cooperating elements in order to carry out the process of designing and qualifying products.

The performed cognitive tests and numerical analyses showed the validity of the adopted process of proceeding in the scope of evaluation of phenomena occurring in highly stressed elements produced from materials with degressive-progressive nonlinear deformation characteristics.

The research confirmed that the behaviour of hyper-elastic material is influenced by a number of phenomena, including in particular the Mullins effect, which manifested itself in the change of stiffness of the material under the influence of successive cycles of deformation.

The developed numerical model of the buffer, containing a description of the material prepared by the authors, made it possible to map the way the security unit works. The conducted numerical simulations allowed to mark the most stressed areas and revealed the essence of changes in the shape of the element. Based on analyses of numerical tests results it was found that the most significant phenomena occur inside the buffer, and more precisely on the internal cylindrical surface. It was justified that the key influence on the buffer's compression process is the axial-symmetrical hole, 
which stimulates the formation of phenomena resembling local loss of stability, consisting in the change of the compression process into bending. Peripheral cuts only increase buffer predisposition to the above phenomenon.

A concurrent method of design and numerical verification of protective elements possible to be manufactured from hyper-elastic porous materials was developed, which consists of the structural design of the component (system) numerical analysis of the assembly behaviour for critical cases and when it is necessary to identify the material of the experimental study. The issues described in the article, including experimental tests and structural identification of the behavior of elastomeric materials, are an important contribution to the development of a number of technical fields. The authors' experience as well as formulated observations and conclusions from the paper are the basis for design and research-implementation works in the field of developing safety systems for working machines. Because of the problems and issues described in the article: multi-criteria approach to the topic, development of new knowledge in the field of systems construction limiting the effects of failures and accidents, design, material engineering, identification and inference supported by a number of research at the structural and physico-mechanical level of the material, broaden the knowledge on a number of platforms for the exchange of engineering experience fit into the mainstream transdisciplinarity of the conference.

\section{References}

[1] J. Osiński, P. Żach, Symulacyjna ocena możliwości zbudowania lekkiego elementu ochronnego, Problemy Eksploatacji, Zeszyty Naukowe, Instytut Technologii Eksploatacji, Radom, 2002. 3/2002, pp. 17-37.

[2] P. Żach, Damping with hyperelastic composite structures, Monografia Wydawnictwa Politechniki Łódzkiej Dynamical Systems Application, 2013, ISBN 978-83-7283-589-5.

[3] EN 81-20:2014.

[4] J. Osiński, P. Żach, Dynamic Deformation Construction made by Viscoelasticity Materials, 34 Solid Mechanics Conference SOLMECH Zakopane, September 2-7, 2002, Volume of Abstracts, pp. 207-208.

[5] P. Żach, Hyperdeform Polymers Materials Vibration Analyses Set of Machines, 3rd International Conference on Mechanical Engineering and Mechatronics, ICMEM 2014.

[6] P. Żach, Description of phenomena vibration in hyperdeform polymers materials, International Journal of Dynamics and Control, 2015, Vol. 3, Issue 1, pp. 36-40.

[7] P. Żach, Structural identification of elastic-damping properties of hyperdeformable materials, Wydawnictwo Naukowe Instytutu Technologii Eksploatacji - Państwowego Instytutu Badawczego, Radom-Warszawa, 2013.

[8] Ł. Gołębiewski, P. Żach, J. Mańkowski, Numerical analysis of the work of a PUR cellasto material buffer, COMPUTATIONAL TECHNOLOGIES IN ENGINEERING (TKI'2018): Proceedings of the 15th Conference on Computational Technologies in Engineering, AIP Conference Proceedings, Vol. 2078, 2019, AIP Publishing, ISBN 978-0-7354-1806-6, pp. 020025-1 - 020025-6.

[9] I. M. Ward, Mechanical Properties of Solid Polymers, John Wiley\&Sons Ltd, London, New York, 1971.

[10] J.D. Ferry, Viscoelastic Properties of Polymers, 3rd ed., John Wiley, New York, 1980.

[11] M. Mooney, A theory of large elastic deformation, Journal of Applied Physics, 1940, 11(9), str. 582592.

[12] R. S. Rivlin, Large elastic deformations of isotropic materials III. Some Simple problems In cylindrical polar coordinates, Philosophical Transactions of the Royal Society of London, Series A 240, 1948, pp. 509-525.

[13] R. S. Rivlin, Large elastic deformations of isotropic materials. IV Further developments of the general theory, Philosophical Transactions of the Royal Society of London. Series A 241, Mathematical and Physical Sciences, 1948.

[14] P. J. Blatz, W. L. Ko, Application of Finite Elastic Theory to the Deformation of Rubbery Materials, Transactions of the Society of Rheology, 1962, Vol. 6, pp. 223-251. 
[15] R.W. Ogden, Large Deformation Isotropic Elasticity - On the Correlation of Theory and Experiment for Incompressible Rubberlike Solids, Proceedings of the Royal Society of London 1972, Vol. 328, Issue 1575 .

[16] O. H. Yeoh, Some forms of the strain energy function for rubber, Rubber Chemistry and Technology, 1993, Vol. 66, pp. 754-771.

[17] O. H. Yeoh, Phenomenological Theory of Rubber Elasticity, Comprehensive Polymer Science, Ed. SL Aggarwal, 2nd Supplement, Pergamon Press 1995.

[18] E. M. Arruda, M. C. Boyce, A three-dimensional constitutive model for the large stretch behavior of rubber elastic materials, 41 N2, Journal of the Mechanics and Physics of Solids, 1993, pp. 389-412.

[19] A. N. Gent, A new Constitutive Relation for Rubber, Rubber Chemistry and Technology, 1996, Vol. 69, pp. 59-61.

[20] M. Dudziak, J. Mielniczuk, Nieklasyczne modele materiałów w projektowaniu maszyn, Wydawnictwo Instytutu Technologii Eksploatacji, Radom 2001.

[21] M. Schrodt, G. Benderoth, A. Kuhhorn, G. Silber, Hyperelastic Description of Polymer Soft Foams at Finite Deformations, TECHNISCHE MECHANIK, Band 25, Heft 3-4, 2005, pp. 162- 173.

[22] A.A. Siranosian, R.R Stevens, Developing an Abaqus *HYPERFOAM Model for M9747 (4003047) Cellular Silicone Foam (Los Alamos National Lab. (LANL), Los Alamos, NM (United States), 2012)o, Radom-Warszawa, 2013.283-589-5. 